\title{
A Study on the Teaching of Lianmian Words in International Chinese Education
}

\author{
Zhang Junjiao ${ }^{1, a}$,Qi Juanli ${ }^{2, b^{*}}$ \\ ${ }^{I}$ Department of Humanities and Management, Shaanxi University of Chinese Medicine, Qindu, Xianyang, Shaanxi, \\ China \\ ${ }^{2}$ Department of Literature and Communication, Xianyang Normal University, Weicheng, Xianyang, Shaanxi, China \\ ajj90219@qq.com \\ b*qijunali2009@126.com
}

\begin{abstract}
Lianmian words is a special type of Chinese vocabulary. This paper combines the current situation and existing problems of the research on the teaching of Lianmian words in international Chinese education.Based on the current situation and existing problems in the teaching and research of Lianmian words in international Chinese education, this paper holds that Lianmian words can be explained from the relationship between their meanings and symbols, the characteristics of their initials and rhymes, and the historical and cultural informa tion contained in them, so as to promote Chinese learners'understanding of Lianmian words and their absorption of Chinese traditional culture.In order to make a breakthrough in the teaching method of Lianmian words in international Chinese education.
\end{abstract}

Keywords: Lianmian word,Chinese education, Culture

\section{国际中文教育联绵词教学研究}

\author{
张隽姣 ${ }^{1,}$, 开娟莉 ${ }^{2,}$ b* \\ ${ }^{1}$ 陕西中医药大学人文管理学院, 秦都, 咸阳, 陕西, 中国 \\ 2 咸阳师范学院文学与传播系, 渭城, 咸阳, 陕西, 中国 \\ azjj90219@qq.com \\ $b^{*}$ qijunali2009@126.com
}

\section{摘要}

联绵词是汉语词汇中一种特殊的类型。本文结合国际中文教育联绵词教学研究现状及存在的问题, 认为在教学 中可以从联绵词与其字义及义符的关系，并运用其声韵的特点，再结合联绵词所蕴含的历史文化信息加以讲 解, 以促进汉语学习者对联绵词的理解以及对中国传统文化的吸收, 以期在国际中文教育中联绵词的教学方法 方面有所突破。

关键词：联绵词，中文教育，文化

\section{1. 联绵词概述}

联绵词, 又写作 “联绵字” “连绵词” “连绵字” 等。黄伯荣、廖序东在《现代汉语》中对联绵词的定 义为：“联绵词指两个不同的音节连缀成一个语素, 表示一个意义的词。” ${ }^{[1]}$ 周荐在《现代汉语词汇学教 程》中解释为: “联绵词又称为 “化石词”, 是单字 词汇单位向复字词汇单位转化过程中的产物, 是单字 词汇单位向复字词汇单位过渡的桥梁和中介。” ${ }^{[2]}$ 赵
克勤在《古代汉语词汇学》中认为 “连绵字是由只代 表音节的两个汉字组成的表示一个整体意义的双音 词。”其中更强调联绵词的三个要点：必须由两个汉 字组成; 必须是单语素; 两个汉字都只起表音作用, 没有意义。 ${ }^{[3]}$ 谢纪锋认为联绵词最基本的特点就是把 两个字当作一个语言单位来训释而非把两个字拆开 来讲。 ${ }^{[4]}$ 简单来说联绵词就是双音节单纯词。本文举 例的联绵词不包含属于单纯词的叠音词。 
虽然普遍认为组成联绵词的两个汉字只是表音 作用，但在联绵词的发展过程中，一些与词义相关的 字被固定下来，而纯粹表音的字被逐渐淘汰。例如“匍 匐” 是联绵词, 义为手足伏地爬行, 又可以写作 “蒲 伏” “伏服” “蒲服” 等, 因《说文解字》中 “匍” 有爬行义, “匐” 有伏地义, 两者都与词义有关, 而 逐渐成为了最普遍的写法, 随着文字的规范化进程, 这些与词义相近的字, 就被固定为标准写法, 也就使 得部分联绵词中的字义并不是与词义毫无联系, 这为 现在的联绵词教学提供了一定便利。在联绵词中, 有 的字本身没有单独的意义也就没有与词义相联系的 意义, 如: 鹦武; 有的是其中一个字有单独的与词义 相联系的意义, 如: 逍遥; 还有一种是两个字都有与 词义相联系的意义, 如: 琳琅。在联绵词的发展中, 也发生了同化现象, 很多词的字, 偏旁相同, 如前例 中, “鹦武” “逍遥” “琳琅”。通过观察, 可以发 现, 这些义符大都具有表意的作用, 且所表现的意义 与词义有一定联系。从某种程度上来说, 这些义符对 联绵词的教学有辅助作用。

所以, 笔者认为, 在联绵词产生初期, 记录联绵 词的字并不固定, 确实只是表音作用, 但在经历了长 时间的发展和文字规范化之后, 部分固定下来的记录 联绵词的字已经不仅仅是表音的作用, 其字义也与联 绵词词义有了联系。

\section{2. 国际中文教育联绵词教学研究现状及难点 分析}

国际中文教育词汇教学研究方面对联绵词教学 的研究近年来也有新的进展。潘燕在《《博雅汉语 $\rangle$ 中 联绵词及相关的复合词研究》中, 以《博雅汉语》等 教材为语料基础, 通过对联绵词及相关复合词的语义 关联的探究展开联想教学, 探索国际中文教育中联绵 词的教学方法。 ${ }^{[5]}$ 刘千楚在《多义联绵词的对外汉语 教学研究》中, 着重研究了多义联绵词, 并通过对联 绵词字义及词义的联系以及多义联绵词多个义项脉 络的分析, 探究用联想和引申等方式进行教学。 ${ }^{[6]}$

一般来说, 在进行词汇教学时, 教师会遵循汉语 词汇教学的原则, 相对重视语素教学, 词与字教学相 结合。但联绵词与复合词不同, 它具有自己的特殊性。

从字义和词义的角度来看, 在联绵词中, 两个汉 字只是代表两个音节, 两个汉字合起来, 才代表一个 词, 这会出现两个难点。一方面, 对于教师来说, 如 果不能意识到这个词是联绵词, 而将其当做复合词, 用一般复合词的教学方法, 把词拆分为字, 从字义教 学入手, 就很可能产生错误的教学, 如 “望洋” 并非 望着江河的意思, 而是仰视的意思; 另一方面, 对于 教师来说, 在教学的时候, 认识到教学内容是联绵词, 可能一味地认为字与词之间只有表音的关系, 而简单 地将联绵词作为一个整体进行教学, 忽略了联绵词中 的字义或字的义符与联绵词词义之间的联系。总体来 说, 如果教师对联绵词以及联绵词与文字之间的联系
把握不到位，就容易造成联绵词教学的困难。

从语音的角度来看, 联绵词大多为双声叠韵, 这 在母语者看来, 比较有古典韵味, 在教学方面, 一定 程度上可以减轻学习者对词汇的语音记忆的负担, 但 对于部分二语学习者来说, 在发音方面会有较大的困 难, 尤其是部分叠韵词, 如 “翩距” 等, 在发音的时 候, 因为对拼音的拼读不熟悉等原因, 容易一带而过 而将第二个字的声母和韵头吞掉。还有一些叠韵词, 两个字中有的有韵头, 有的没有韵头, 学习者很容易 将两者都读作有韵头或无韵头, 以至于出现语音错误。 如: 彷得。所以, 这些情况, 都需要教师带领学习者 进行反复练习并加以强调以避免错误。

\section{3. 联绵词音、义教学方法的探究}

就字、词意义而言, 从意义的角度, 联绵词虽然 表示一个语素，但并不是所有的联绵词词义与字义之 间都毫无关联。相当大部分的联绵词中两个字的意思 与该联绵词的意义是有关联的, 可以采用讲解字的意 义和联绵词意义之间的联系来进行教学。还有部分联 绵词, 虽然其字义与词义没有联系, 但在字形上, 其 二字义符相同, 且与联绵词的词义有一定的联系。

从语音的角度, 联绵词中双声叠韵很多, 可以强 调声母或韵母相同, 来增强学习者的记忆。在讲解联 绵词的时候, 要注重归纳, 首先可以引导学习者自己 思考联绵词中两个字的读音的规律, 当学习者自己归 纳出来时, 其印象更为深刻, 也能提升其汉语学习的 成就感。

笔者认为, 在联绵词的教学中可以首先分析联绵 词义和其字义之间是否有联系, 以决定是整体教学还 是从字义入手进行教学。其次看字形中的义符是否与 词义有关, 若是有关, 则可以义符的意义辅助学习者 理解词义。最后再从语音入手，音义结合进行教学。 下面笔者将以双声词 “枇杷” “琳琅” “含糊”，叠 韵词 “从容” “彷得” “逍遥” , 非双声叠韵词 “鹦 鹉” “牡丹” “滂沱” “当归” 为例进行具体的教学 分析。本文分析的联绵词只取其联绵词义中最常用的 一个意义。字义取与联绵词联系最紧密的意义。

枇杷: 植物名, 果实可食。枇: 枇杷。杷: 一种 有齿和长柄的农具。从意义来看, “枇” 没有意义, “杷” 与词义并无联系, 需要进行整体的教学。“枇” “杷”二字都有义符 “木”，“木”表植物，在教授 该词时, 可以双声结合义符的方式进行教学。首先, 通过图片展示, 让学习者直观而形象地了解枇杷的意 义。而后从语音方面, 着重强调两字声母相同, 让学 习者针对声母进行反复练习, 使其更好地掌握读音。 最后, 从义符 “木” 入手, 讲解两字都有 “木” , 表 示这个词和植物有关, 便于学习者记忆。在讲解 “枇 杷” 读音时, 需注意该词两个字的声符 “比” “巴” 均与本字读音不同, 要警惕学习者混淆读音。

琳琅：玉石撞击的声音。琳：美玉。琅：似珠的 
美石。从意义来看, “琳” “琅” 字义与词义都有联 系，可以从字义联系词义进行教学。“琳” “琅”二 字都有义符“王”, 表玉器。在教授该词时, 可以双 声结合字义、义符的方式教学。首先, 讲解“琳” “琅” 二字都有玉石之义, 进而引导出 “琳琅” 的玉器撞击 之义。从语音方面, 着重强调两字声母相同, 让学习 者针对声母进行反复练习, 使其更好地掌握读音。在 讲解“琳琅”读音时, 也尽量避免因“琅”的声符“良” 与其不同音而造成错读。最后, 从义符“王” 入手, 讲解两字都有 “王”, 表示这个词和玉器有关, 便于 学习者记忆。

含糊: 声音、言语或意思不清晰。含: 衔在嘴里, 不吐出也不咽下。糊: 粥类食品。从意义来看, “含” “糊” 字义与词义没有联系, 但可以用联想的方式讲 解, 因为如果嘴里喊着一口粥, 就会言语不清晰, 从 字义联系词义进行教学。首先, 通过播放小短片, 展 示出 “含糊” 的意思, 再讲解 “含” “糊” 可以联想 到嘴里含着粥导致言语不清晰, 让学习者进行联想记 忆。这样的教学并不是认为 “含” “糊” 的字义与词 义有联系, 只是采用联想的方式, 增强学习者对该词 的理解和记忆。语音方面, 要着重强调 “含糊” 是双 声词, 切忌不可将第二个字的声母吞掉。

从容: 不慌不忙, 悠闲舒缓的样子。从: 跟随, 依顺。容: 容貌, 仪表, 景象, 状态。“从” 有多个 意义但其字义和词义没有联系, “容” 的多个意义中 “容貌状态”之义和词义有一定联系, 可以适当讲解 “容” 的意思, 使学习者可以进行联想记忆。构成该 词的两个字没有相同的义符, 可以叠韵结合单字“容” 的意义讲解。“从容” 属于稍显抽象的形容词, “从 容” 的文字没有义符, 需要通过讲解对比词, 如 “慌 张” 等。通过表演的方法, 采用对比的方式, 先展示 “慌张” 的状态, 对比展示出 “从容” 的意义。在此 基础上, 可以适当讲解 “容” 的 “状态” 义, 使学习 者牢记 “从容” 是一种状态。讲授语音的时候着重强 调两个字的韵母相同, 通过不断的练习巩固学习者的 发音, 在发叠韵的音时, 要尽量让学习者将两个字的 音发清楚, 避免出现第二个字的声母被吞的情况。

彷得: 徘徊, 犹豫不决。彷: 彷得。徨: 彷得。 “彷” “得” 两个字没有意义, 只能进行整体教学。 “彷” “得”二字都有义符 “㣙”, 多与行走有关。 教授该词时, 可以叠韵结合义符的方式讲解。首先通 过表演走来走去, 犹豫不决的样子, 解释 “彷得” 的 意义。进行教学时, 通过表演的方式引出 “彷得” 的 词义。讲授语音的时候着重强调两个字的韵母相同, 通过不断的练习巩固学习者的发音, 在发叠韵的音时, 要着重让学习者体会“得”的韵头的发音, 避免吞音, 也要避免声母被吞音出现没发出来的情况。最后从义 符 “㣙手, 讲解 “彷得” 与走来走去有关, 引申 走来走去是犹豫不决的样子。

逍遥: 自由自在、不受拘束。逍: 逍遥。遥: 远。 从字义来看, “逍” 没有单独的字义, “遥” 有单独
的意义但与词义没有联系, 只能进行整体教学。“逍” “遥”二字都有义符 “之”，表走走停停之义。在教 授该词时, 可以叠韵结合义符的方式进行讲解。“逍 遥” 属于稍显抽象的形容词, 可以展示对比词, 如 “拘 谨” 等来辅助理解, 通过展示图片对比出拘谨不自由 的状态和逍遥自在的状态。讲授语音的时候着重强调 两个字的韵母相同, 通过不断的练习巩固学习者的发 音, 在发叠韵的音时, 要着重让学习者体会韵头的发 音, 避免出现吞音。最后从义符“之” 入手, 讲解 “逍 遥” 走走停停, 随心所欲, 是一种自由自在不受拘束 的表现。

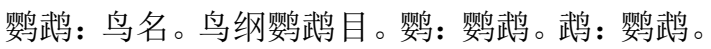
从意义来看, 字义与词义没有联系, 需要进行整体教 学。“鹦” “陚”二字都有义符 “鸟”, “鸟” 表鸟 类。在教授该词时, 可以结合义符教学。和 “枇杷” 类似, 通过展示鹦武的图片, 让学习者产生联想记忆, “鹦鹉” 的语音虽然非双声叠韵, 但 “鹦鹉” 的读音 和鸟鸣类似, 可以通过拟声的方, 引导学习者, 加强 其对读音的记忆。最后, 通过义符 “鸟”, 巩固学习 者的记忆, 让学习者看见字形, 能第一时间想到这个 词和鸟有关。

牡丹: 植物名, 药药属草本植物或灌木, 红色或 白色。牡: 雄性的鸟兽。丹: 红色。牡丹的字义和词 义没有联系, 也不是双声叠韵词, 字形也没有义符和 词义产生联系，但因其是表示具体事物的名词，教师 可以通过展示图片的方式, 讲解牡丹的意义。同时, 在讲解该词时, 可以从 “丹” 的 “红色” 义入手, 牡 丹多为红色, 以此加深学习者的记忆。

浐沦: 雨大的样子。滂: 形容水涌出。沱: 可以 停船的水湾。“滂” 字义与词义有明显联系, “沱” 的字义中“水”之义可以与词义有一定联系。字形上， 二者皆有义符 “今” 表水之义, 可以讲解浐的意思并 辅以两字的义符, 让学习者进行联想记忆。教师在讲 解 “滂沱” 时, 可以先播放一幅下着大雨的动态图片, 让学习者直观感受到 “滂沱” 的意义, 再分别解释两 个字的意思, 最后从字形的义符 “澺”进行讲解, 表 示该词与水有关。

当归: 草本植物, 根入药, 有镇静、补血、调经 等作用。当: 应该, 应当。归: 返回。从意义上看, 当归的字义与词义没有任何联系, 语音方面也是非双 声叠韵词, 字形上也没有与词义相关的义符。在讲解 时教师可以以图片展示的方式进行教学。但这里列出 当归还有一个原因, 就是在这种语音意义和字形都没 有联系的情况下, 还可以尝试用讲解故事的方式进行 教学。古人会在寄给远行之人的信中放上当归, 表示 “你应当回来了” 的思念之情, 加强学习者的记忆。

通过对以上例词的教学分析, 可以看出, 在讲授 联绵词时, 图片展示法对导入名词性词语尤其是指代 具体事物的名词有促进作用, 而形容词因为其抽象性, 可以采取图片或表演的方式进行。其次, 要注重联绵 词的读音讲解, 联绵词双声叠韵多, 通过语音的讲解 
和反复操练, 可以简化学习者对语音的记忆, 但同时 要避免因双声叠韵造成的吞音现象。第三, 对于字义 和词义有联系的联绵词, 要强调其联系, 对于字义和 词义没有联系的联绵词, 首先需要从字形入手, 如果 字形也与词义没有关联, 还可以从两个字在一起进行 联想的方式入手, 如 “含糊”, 适当采用联想的方法, 可以促进学习者的记忆; 对于字义和词义无联系又不 容易产生正确联想的联绵词, 不要强行拆分成字讲解, 在学习者对字义和词义的联系提出疑惑时, 尽可能让 学习者不要从字义对词义进行联想记忆。对于有简单 而好记的典故的词语, 可以采取讲授典故的方式, 辅 助学习者理解记忆。最后, 要注重汉字字形的教学, 重视联绵词中相同义符的表义作用, 以进行辅助教学。

在文字方面还有一个问题, 就是联绵词中也有部 分形声字。形声字的声符虽然具有一定的表音作用, 但不是所有的字都与其声符读音完全相同, 甚至有一 些字与其声符的读音完全不同。在国际中文教学中, 学习者很难在短时间里区分或记住哪些字与声符读 音完全相同, 哪些只是韵母相同, 哪些仅仅是韵腹+ 韵尾相同, 哪些又是完全不同。所以, 以笔者个人拙 见, 在条件不成熟的情况下, 为了避免学习者通过声 符记音而造成混淆读音出现 “认半边字” 的情况, 尽 量不要轻易从形声字的声符入手进行教学。

\section{4. 联绵词中文化教学的探究}

语言是文化的载体和传播媒介, 在国际中文教育 词汇教学中, 可以搭载物质文化乃至精神文化的教学。 目前研究国际中文教育词汇教学中的文化因素教学 的资料相对多一些, 而研究联绵词的文化因素如何与 国际中文教育结合起来的资料相对较少。笔者认为, 无论是语言教学方面, 还是帮助二语学习者尽快融入 目的语文化方面, 文化教学都有积极的作用。正如赵 德荣在《对外汉语词汇教学中的文化因素及其教学》 中提到的, 对于初级汉语学习者来说, 教师需要教授 学习者一些汉语词义的文化意义, 方便其理解并正确 使用所学词汇。 ${ }^{[7]}$

普遍认为, 大部分联绵词是古代汉语的遗留, 蕴 含了深刻的中国传统文化。在进行联绵词教学时, 可 以将联绵词产生的背景或所涉及的典故一并讲授, 在 加深学习者理解的同时, 也能增加学习者对中国文化 知识的了解。以 “牡丹” 为例, 牡丹作为一种花卉, 对外国学习者或许并没有什么特别之处, 但在中国历 史上, 牡丹的象征意义或远高于其作为一种花卉的观 赏意义。在讲解牡丹时, 教师可以讲解牡丹在李唐时 期是富贵的象征, 引出大唐盛世进而向学习者介绍盛 唐的繁华。也可以讲解牡丹因为天生傲骨不肯屈服于 武则天而被贬洛阳的传说, 引出对人的品格的探讨。 不仅如此, 牡丹作为一种植物, 还具有药用价值, 教 师亦可以从中医的角度, 讲解牡丹的药用价值, 以促 进中医药文化的传播。在讲解 “逍遥” 时, 教师可适 当讲解与 “逍遥” 相关的人物的故事, 如介绍庄子的 作品《庄子 - 逍遥游》并讲授其生平和处事观念, 以
此引出中国道家文化, 与此同时, 可以在此基础上, 介绍更多的中国传统哲学思想如儒家的仁爱、中庸, 墨家兼爱非攻等, 以引导学习者了解并探讨中国传统 的哲学观、处世观, 并对一些问题进行适当讨论, 拓 展文化教学的广度和深度。

正因为联绵词有丰富的历史文化背景, 在词汇教 学中, 可以更好的融入文化因素。通过讲解历史故事、 典故传说, 不仅可以使课堂更具有趣味性, 也能在教 授词汇时通过讲解其所涉及的历史文化背景, 加深学 习者的印象。同时在尊重各国文化的前提下, 适当探 讨中西方文化的差异, 让学习者更深入的了解中国文 化, 逐渐适应中国思维。

\section{5. 结论}

联绵词虽然具有其特殊性，但随着时间的发展和 语言文字的规范，许多联绵词的词义和文字之间有了 一定联系。在讲授联绵词时要充分了解其字义与词义 的关系, 重视义符的表意作用, 结合声韵特征进行语 言教学。同时充分挖掘联绵词背后的文化因素, 通过 对其所包含的历史典故的讲解, 将文化因素融入词汇 教学之中。在进行语言教学的基础上, 实现文化教学 的目的。

\section{REFERENCES}

[1] Huang,B.R.,Liao,X.D.(2011) Modern Chinese. Higher Education Press,Beijing.

[2] Zhou,J.(2016) Modern Chinese Lexicology Course. Peking University Press, Beijing.

[3] Zhao,K.Q.(1994) Ancient Chinese lexicology. The Commercial Press, Beijing.

[4] Xie,J.F.(2011) Dictionary of Chinese Lianmian Word. Foreign Language Teaching and Research Press.Beijing.

[5] Pan,Y. (2019) A Study of Lianmian Words and Related Compounds .Hunan University.

[6] Liu,Q.C.(2019)The Research on Teaching Chinese as a Foreign Language in Multi-sense Joint Words.Hunan University.

[7] Zhao,D.R.(2013) Cultural Factors of Vocabulary Teaching in Teaching of Chinese as A Second Language.Hunan University.

[8] Liu,X.(2009) An Introduction to the Pedagogy of Teaching Chinese as a Foreign Language. Beijing Language and Culture University Press, Beijing. 